\title{
Review: placebo is better than no treatment for subjective continuous outcomes and for treatment of pain
}

Hróbjartsson A, Gøtzsche PC. Is the placebo powerless? An analysis of clinical trials comparing placebo with no treatment. N Engl J Med 2001 May 24;344:1594-602.

QUESTIONS: In patients with various clinical conditions, what is the clinical effect of placebo as a treatment for disease? Does the effect differ for subjective and objective outcomes?

\section{Data sources}

Trials published before the end of 1998 were identified by searching Medline, EMBASE/Excerpta Medica, PsycLIT, Biological Abstracts, and the Cochrane Controlled Trials Register. The search terms were synonyms of placebo, no treatment, and randomised clinical trial. Reference lists of included trials and selected review articles and books were reviewed, and experts were contacted.

\section{Study selection}

Studies were selected if patients were randomly allocated to a placebo group or an untreated group. Exclusion criteria were unconcealed randomisation, sample of paid or healthy volunteers, non-blinding of assessors of objective outcomes, $>50 \%$ dropouts, or if the alleged placebo had a clinical effect not associated with the treatment ritual alone.

\section{Data extraction}

Data were extracted using predetermined forms. Primary outcome of interest was the main outcome defined by the trial author or if not defined, the most clinically relevant outcome.

\section{Main results}

114 trials published from 1946-98 were included in the analysis. The typical placebo for drugs was a lactose tablet; for physical interventions, the procedure done with a machine turned off; and for psychological interventions, an attention placebo. 40 clinical conditions were investigated.

Meta-analysis showed that placebo did not differ from no treatment for binary outcomes (32 trials, $\mathrm{n}=3795$ ), regardless of whether outcomes were subjective (23 trials, $\mathrm{n}=1928$ ) or objective ( 9 trials, $\mathrm{n}=1867)$; significant heterogeneity existed among trials $(\mathrm{p}=0.003)$. Placebo did not differ from no treatment in trials assessing nausea (3 trials, $\mathrm{n}=182)$, smoking cessation relapse ( 6 trials, $\mathrm{n}=887$ ), or depression ( 3 trials, $\mathrm{n}=152$ ).

Meta-analysis showed that placebo was more effective than no treatment for continuous outcomes overall (82 trials, $\mathrm{n}=4730$ ) and for subjective continuous outcomes only (53 trials, $\mathrm{n}=3081$ ) (table); placebo did not differ from no treatment when considering objective continuous outcomes only (29 trials, $n=1649)$. Significant heterogeneity existed among trials $(\mathrm{p}<0.001)$, and magnitude of placebo effect decreased with increasing sample size $(\mathrm{p}=0.05)$. Of trials assessing continuous outcomes, placebo was more effective than no treatment for pain (27 trials, $\mathrm{n}=1602$ ) (table), but not for obesity
( 5 trials, $\mathrm{n}=128$ ), asthma ( 3 trials, $\mathrm{n}=81$ ) hypertension (7 trials, $\mathrm{n}=129)$, insomnia ( 5 trials, $\mathrm{n}=100$ ), or anxiety (6 trials, $\mathrm{n}=257)$.

\section{Conclusions}

Placebo was more effective than no treatment in trials assessing continuous subjective outcomes and in trials assessing treatment of pain. Placebo and no treatment did not differ in trials assessing objective or subjective binary outcomes or objective continuous outcomes.

Placebo v no treatment for various conditions* Outcomes at the end of treatment Pooled standardised mean difference $(95 \% \mathrm{Cl}) \dagger$

\begin{tabular}{ll} 
Overall continuous outcomes & $-0.28(-0.38$ to -0.19$)$ \\
\hline Subjective continuous outcomes & $-0.36(-0.47$ to -0.25$)$ \\
\hline Pain (continuous outcomes only) & $-0.27(-0.40$ to -0.15$)$ \\
\hline${ }^{*}$ Abbreviations defined in glossary. &
\end{tabular}

†Negative value indicates a beneficial effect of placebo.

\section{COMMENTARY}

This meta-analysis by Hróbjartsson and Gøtzsche is controversial both theoretically and practically. Theoretical difficulties may be highlighted by the fact that the very authors of this epoch making meta-analysis once argued that placebo is undefinable ${ }^{1}$ and that the placebo effect is uncontrollable (ie, any comparison would underestimate it). ${ }^{2}$ The all sweeping nature of the conclusions in the original paper is also questionable: a meta-analytic summary is justified insofar as a more or less similar magnitude of effect can be expected across the range of patients, the range of interventions, and the range of outcomes for the included studies. May we expect such similar effects of comparisons of placebo and no treatment for smoking, nausea, infertility, infection, Parkinson's disease, etc? In my opinion, the abstract above, independently prepared by the editorial office of this journal, is a better summary.

Practically then, it appears wise to concentrate on the substantive areas in which practising clinicians may be interested. For pain, the pooled standardised mean difference (SMD) was -0.27 ( $95 \%$ CI -0.40 to -0.15$)$; the point estimate suggests a small effect, but the $95 \% \mathrm{CI}$ is such that one cannot rule out the possibility of a moderate effect. For insomnia, the SMD was -0.26 (CI -0.66 to 0.13 ); for anxiety, it was -0.06 , (CI -0.31 to 0.18 ). The confidence intervals are too wide to rule out a large to moderate effect. For all the other clinical questions within mental health, $\leqslant 3$ RCTs were available.

On the basis of the present state of knowledge, we cannot claim that we could use "placebo treatment"for depression or anxiety. ${ }^{3}$ Nor should we talk about maximising placebo effects in our treatment unless good evidence exists to show that we really can do so. ${ }^{4}$ We are still in need of more research on placebo effects within mental health. Toshi A Furukawa, MD, PhD Nagoya City University Nagoya, Japan

1 Gøtzsche PC. Is there logic in the placebo? Lancet 1994;344:925-6.

2 Hróbjartsson A. The uncontrollable placebo effect. Eur J Clin Pharmacol 1996;50:345-8.

3 Enserink M. Can the placebo be the cure? Science 1999;284:238-40.

4 Kleijnen J, de Craen AJ, van Everdingen J, et al. Placebo effect in double-blind clinical trials: a review of interactions with medications. Lancet 1994;344:1347-9. 